\section{Nuclear Medicine Training: Two Different Pathways?}

TO THE EDITOR: I read with interest the editorial written by Segall et al. (1). The authors discuss a combined, multispecialty training "that maintains high standards for nuclear medicine education." A 3-y residency in nuclear medicine that leads to American Board Nuclear Medicine certification alone may "not provide diplomates with adequate employment opportunities." Instead, the proposed combined training would require 16 mo for nuclear medicine and 32 mo for radiology.

We do not believe that it is possible to provide a solid nuclear medicine training program in only $16 \mathrm{mo}$; the same holds true for the proposed 32-mo radiology program. This becomes evident when the contents of the full training programs are considered.

For example, radiology training must cover the topics of CT (thorax, abdomen, and angiography), neuro-CT, MRI (including of the brain), interventional radiology, ultrasound (vascular, pediatric, gynecologic, urologic, orthopedic, and trauma), and conventional radiology (thorax, abdomen, trauma, orthopedics, endocrinology, and surgery).

Similarly, nuclear medicine training must include PET/CT, SPECT/CT, neuro nuclear medicine, endocrinology (thyroid and others), radiation biology, radiophysics, radiochemistry, and radiation safety. It must also cover "bread and butter" nuclear medicine, including bone, myocardium, lung, renal (genitourinary tract), liver (hepatobiliary), and radionuclide therapies (the latter of which has gained importance in the treatment of various malignancies). Additionally, the increasingly important topic of nuclear oncology could become a subspecialty of nuclear medicine, as has been suggested by Ahmadzadehfar and Essler (2).

From this summary, it appears evident that it is not possible to provide comprehensive training in nuclear medicine and radiology within a short 4-y program. In Germany, a similar program leading up to the 2 board certifications requires a total $8.5 \mathrm{y}$ of study. As this is a much longer period than the one proposed by Segall et al. (1), we believe that 2 independent training programs are necessary to adequately train nuclear medicine and radiology residents (3).

Instead of seesawing between different specialties, we should accept nuclear medicine as a full specialty in its own right. Its need for CT to better anatomically localize its findings does not mean that this specialty should fall under the banner of radiology. Similarly, the presence of radionuclide therapies in nuclear medicine does not relegate it to the field of radiation oncology. The addition of sectional imaging training to nuclear medicine residency programs has the potential to solve all of these problems. This does not mean that a nuclear medicine physician will report CT or MRI, but SPECT/PET/CT and PET/MRI can be reported by nuclear medicine physicians.

\section{REFERENCES}

1. Segall GM, Grady EE, Fair JR, Ghesani MV, Gordon L. Nuclear medicine training in the United States. J Nucl Med. 2017;58:1733-1734.

2. Ahmadzadehfar H, Essler M. It is time to move forward into the era of theranostics. EJNMMI Res. 2018;8:9.

3. Biersack HJ. PET-CT: comments on the white paper. Eur J Nucl Med Mol Imaging. 2008;35:1576.

Hans Jürgen Biersack
University Hospital Bonn
Sigmund-Freud-Strasse 25
53127 Bonn, Germany
Email: hans-juergen.biersack@ukb.uni-bonn.de

Published online Mar. 15, 2018. DOI: 10.2967/jnumed.118.210401 